PROCEEDINGS OF THE

AMERICAN MATHEMATICAL SOCIETY

Volume 125, Number 2, February 1997, Pages 477-484

S 0002-9939(97)03571-5

\title{
SMOOTH EXHAUSTION FUNCTIONS IN CONVEX DOMAINS
}

\author{
ZBIGNIEW BŁOCKI \\ (Communicated by Jeffrey B. Rauch)
}

\begin{abstract}
We show that in every bounded convex domain in $\mathbb{R}^{n}$ there exists a smooth convex exhaustion function $\psi$ such that the product of all eigenvalues of the matrix $\left(\partial^{2} \psi / \partial x_{j} \partial x_{k}\right)$ is $\geq 1$. Moreover, if the domain is strictly convex, then $\psi$ can be chosen so that every eigenvalue is $\geq 1$.
\end{abstract}

\section{INTRODUCTION}

Let $D$ be a bounded domain in $\mathbb{R}^{n}$. A function $\psi$ we call a convex exhaustion in $D$ if $\psi$ is convex, negative in $D$ and $\lim _{x \rightarrow \partial D} \psi(x)=0$. One can show that $D$ is convex if and only if it admits a convex exhaustion (see e.g. [Hör, pp. 57-59]). Perhaps the simplest example of an exhaustion in a bounded convex domain $D$ containing the origin is $p_{D}-1$, where $p_{D}$ is the Minkowski functional of $D$. For a larger class of exhaustions see Theorem 4.1.

The goal of this paper is to show the existence of smooth $\left(\mathrm{C}^{\infty}\right)$ convex exhaustions with additional properties in arbitrary convex and strictly convex domains. Recall that a domain is called strictly convex if it is convex and its boundary contains no line segment. Our main results are the following two theorems.

Theorem A. If $D$ is a convex, bounded domain in $\mathbb{R}^{n}$ then there exists a smooth exhaustion $\psi$ in $D$ such that

$$
M \psi:=\operatorname{det}\left(\frac{\partial^{2} \psi}{\partial x_{j} \partial x_{k}}\right) \geq 1
$$

(that is, the product of all eigenvalues of the matrix $\left(\partial^{2} \psi / \partial x_{j} \partial x_{k}\right)$ is $\geq 1$ ).

Theorem B. A bounded domain $D$ in $\mathbb{R}^{n}$ is strictly convex if and only if there exists a smooth convex exhaustion $\psi$ in $D$ such that

$$
\sum_{j, k=1}^{n} \frac{\partial^{2} \psi}{\partial x_{j} \partial x_{k}} y_{j} y_{k} \geq|y|^{2}, \quad y=\left(y_{1}, \ldots, y_{n}\right) \in \mathbb{R}^{n}
$$

(that is, every eigenvalue of the matrix $\left(\partial^{2} \psi / \partial x_{j} \partial x_{k}\right)$ is $\left.\geq 1\right)$.

Note that nothing about the regularity of the domains is assumed.

The main step in proving Theorems A and B is global approximation of strictly convex functions. We do it in section 1 in a more general context, using the terminology of sheaves. The reason for it is that we are going to work in different classes

Received by the editors March 27, 1995 and, in revised form, August 14, 1995.

1991 Mathematics Subject Classification. Primary 26B25; Secondary 35J60.

The author was partially supported by KBN Grant No. 2 PO3A 05809.

(C)1997 American Mathematical Society 
of convex functions and we want to have good approximation by smooth elements in every one of them. Since the methods are similar to those from [Rich] where the case of plurisubharmonic functions was considered, we introduce a notion of a Richberg sheaf.

Having the results of section 1 , it is not difficult to prove Theorem B, since appropriate will then be the sheaf of all strictly convex functions. The proof of Theorem A requires more care, for we shall make use of the theory of the MongeAmpère operator $M$. Without it we can only get a smooth strictly convex exhaustion in Theorem A (that is, $M \psi>0$ instead of $M \psi \geq 1$ ).

Results corresponding to Theorems A and B in the complex case were obtained in $[\mathrm{KR}],[\mathrm{Sib}]$, and $[\mathrm{Bło}]$.

\section{Global approximation, Richberg sheaves}

Let $u$ be a continuous function in an open subset $\Omega$ of $\mathbb{R}^{n}$. Then $u$ can locally be approximated by its regularizations

$$
u_{\delta}(x):=\left(u * \rho_{\delta}\right)(x)=\int_{B} u(x-\delta y) \rho(y) d \lambda(y),
$$

where $\lambda$ is the Lebesgue measure, $B$ the unit ball in $\mathbb{R}^{n}, \delta>0$, while $\rho \in \mathrm{C}_{0}^{\infty}\left(\mathbb{R}^{n}\right)$ is nonnegative, $\operatorname{supp} \rho=\bar{B}, \int_{B} \rho d \lambda=1$ and $\rho_{\delta}(y):=\delta^{-n} \rho(y / \delta)$. Then $u_{\delta} \in \mathrm{C}^{\infty}\left(\Omega_{\delta}\right)$, where $\Omega_{\delta}:=\{x \in \Omega: \operatorname{dist}(x, \partial \Omega)>\delta\}$, and $u_{\delta}$ tends locally uniformly to $u$ as $\delta \downarrow 0$.

More generally, we shall consider functions of the following form:

$$
u_{\theta}(x):=u_{\theta(x)}(x)=\int_{B} u(x-\theta(x) y) \rho(y) d \lambda(y), \quad y \in \Omega_{\delta},
$$

where $\theta \in \mathrm{C}^{\infty}(\Omega), 0 \leq \theta \leq \delta$. Observe that if $u$ is smooth on an open $D \subset \Omega$ then so is $u_{\delta}$ on $D_{\delta}$, for we can then differentiate under the sign of integration.

Definition. A subsheaf $\mathcal{S}$ of the sheaf of continuous functions over $\mathbb{R}^{n}$ is called a Richberg sheaf if the following conditions are satisfied.

(1.1) For any $u \in \mathcal{S}(\Omega), \varphi \in \mathrm{C}_{0}^{\infty}(\Omega)$ and $c \in \mathbb{R}$ there exists $\varepsilon_{0}>0$ such that $u+\varepsilon \varphi+c \in \mathcal{S}(\Omega)$ for $\varepsilon \in\left[0, \varepsilon_{0}\right]$.

(1.2) If $u, v \in \mathcal{S}(\Omega)$ then $\max \{u, v\} \in \mathcal{S}(\Omega)$.

(1.3) If $\Omega^{\prime} \Subset \Omega, \theta \in \mathrm{C}^{\infty}(\Omega), 0 \leq \theta \leq 1$ and $u \in \mathcal{S}(\Omega)$ is smooth on a neighborhood

of $\overline{\{\theta<1\}} \cap \overline{\Omega^{\prime}}$, then $u_{\delta \theta} \in \mathcal{S} \cap \mathrm{C}^{\infty}\left(\Omega^{\prime}\right)$ for $\delta>0$ small enough.

The condition (1.3) means essentially that a Richberg sheaf is closed under the operation of regularization in the generalized sense. It implies in particular (1.3)' If $\Omega^{\prime} \Subset \Omega$ and $u \in \mathcal{S}(\Omega)$, then $u_{\delta} \in \mathcal{S} \cap \mathrm{C}^{\infty}\left(\Omega^{\prime}\right)$ for $\delta$ small enough.

(We assume that the empty set is a neighborhood of itself.)

We want to prove the following:

Theorem 1.1. Suppose $\mathcal{S}$ is a Richberg sheaf. Let $\Omega$ be open in $\mathbb{R}^{n}$ and let $\varepsilon>0$ be a continuous function on $\Omega$. Then for $u \in \mathcal{S}(\Omega)$ one can find $v \in \mathcal{S} \cap C^{\infty}(\Omega)$ such that $u \leq v \leq u+\varepsilon$.

The proof of Theorem 1.1 relies on the following fact.

Lemma 1.2. Let $u \in \mathcal{S}(\Omega)$, where $\mathcal{S}$ is a Richberg sheaf and $\Omega$ an open subset of $\mathbb{R}^{n}$. Assume that $u$ is smooth on a neighborhood of $\bar{D}$, where $D \Subset \Omega$ is open. Let $V$ and $W$ be open such that $V \Subset W \Subset \Omega$, and let $\varepsilon>0$ (constant). Then there exists $v \in \mathcal{S}(\Omega)$ such that 
i) $v=u$ on $\Omega \backslash W$,

ii) $u \leq v \leq u+\varepsilon$ on $\Omega$,

iii) $v$ is smooth on a neighborhood of $\bar{D} \cup \bar{V}$.

Lemma 1.2 easily implies Theorem 1.1:

Proof of Theorem 1.1. Suppose $\Omega_{k} \uparrow \Omega$ where the sets $\Omega_{k} \Subset \Omega_{k+1} \Subset \Omega, k \geq 0$, are open, $\Omega_{0}=\emptyset$. For $k \geq 1$ set $W_{k}:=\Omega_{k+1} \backslash \bar{\Omega}_{k-2}\left(W_{1}:=\Omega_{2}\right)$ and let $V_{k}$ be open such that $\Omega_{k} \backslash \Omega_{k-1} \Subset V_{k} \Subset W_{k}$. Let $\gamma_{k}>0$; it will be specified later. From Lemma 1.2 we can get a sequence $\left\{u_{k}\right\} \subset \mathcal{S}(\Omega)$ such that $u_{0}=u$ and

$$
\begin{gathered}
u_{k}=u_{k-1} \text { on } \Omega \backslash W_{k}, \\
u_{k-1} \leq u_{k} \leq u_{k-1}+\gamma_{k} \text { on } \Omega, \\
u_{k} \text { is smooth in a neighborhood of } \bigcup_{j=1}^{k} \bar{V}_{j}
\end{gathered}
$$

$\left(D=\bigcup_{j=1}^{k-1} V_{j}\right)$. The sequence $\left\{u_{k}\right\}$ is locally constant for $k$ big enough; thus we may define $v:=\lim u_{k} \in \mathcal{S} \cap \mathrm{C}^{\infty}(\Omega)$. Then $u \leq v$ on $\Omega$, and for $x \in \Omega \backslash \Omega_{k}$ one has

$$
v(x)=u(x)+\sum_{j=k}^{\infty}\left(u_{j}(x)-u_{j-1}(x)\right) \leq u(x)+\sum_{j=k}^{\infty} \gamma_{j} .
$$

Now, if $\gamma_{k}$ are such that

$$
\sum_{j=k}^{\infty} \gamma_{j} \leq \min _{\bar{\Omega}_{k+1}} \varepsilon
$$

then $u \leq v \leq u+\varepsilon$ on $\Omega$.

Proof of Lemma 1.2. Let $\eta \in \mathrm{C}_{0}^{\infty}(\Omega)$ be such that $0 \leq \eta \leq 1$ on $\Omega, \operatorname{supp} \eta \subset W$, and $\eta=1$ on a neighborhood of $\bar{V}$.

First assume that $D$ is empty. From (1.1) it follows that there exists $c \in(0, \varepsilon)$ such that $u+c \eta \in \mathcal{S}(\Omega)$. Regularization of $u+c \eta$ and (1.3) give a function $\widetilde{\psi} \in \mathcal{S} \cap \mathrm{C}^{\infty}(W)$ with $|\widetilde{\psi}-u-c \eta| \leq \frac{c}{2}$ on $W$. Define

$$
v:= \begin{cases}\max \left\{u, \widetilde{\psi}-\frac{c}{2}\right\} & \text { on } W, \\ u & \text { on } \Omega \backslash W .\end{cases}
$$

Then $v=u$ if $\eta=0$, and $v=\psi-\frac{c}{2}$ if $\eta=1$. Hence by (1.2), $v \in \mathcal{S}(\Omega), v$ is smooth on a neighborhood of $\bar{V}$, and $u \leq v \leq u+c \leq u+\varepsilon$ on $\Omega$.

Now let $D$ be arbitrary. Choose open sets $G_{j}, D_{j}$ and $\theta_{j} \in \mathrm{C}^{\infty}(\Omega), j=1,2$, so that $0 \leq \theta_{j} \leq 1, \bar{G}_{j}=\left\{\theta_{j}=0\right\}, D_{j}=\left\{\theta_{j}<1\right\}, D \Subset G_{1} \Subset D_{1} \Subset G_{2} \Subset D_{2} \Subset \Omega$ and $u$ is smooth on a neighborhood of $\bar{D}_{2}$. By (1.1) we can find $c \in(0, \varepsilon)$ such that $\widetilde{u}:=u+c \eta \in \mathcal{S}(\Omega)$ and

$$
\widetilde{u}-\frac{c}{2} \theta_{1} \in \mathcal{S}(\Omega)
$$

We claim that there is $\widetilde{\psi} \in \mathcal{S} \cap \mathrm{C}^{\infty}(W)$ such that

$$
\begin{gathered}
\widetilde{\psi}=\widetilde{u} \text { on a neighborhood of } W \cap \bar{D}_{1}, \\
\qquad|\widetilde{\psi}-\widetilde{u}| \leq \frac{c}{2} \text { on } W .
\end{gathered}
$$


Indeed, for $\delta>0$ small enough we have $\left|\widetilde{u}_{\delta}-\widetilde{u}\right| \leq \frac{c}{2}$ on $W$. Thus, for $x \in W$ $\left|\widetilde{u}_{\delta \theta_{2}}(x)-\widetilde{u}(x)\right|=\left|\widetilde{u}_{\delta \theta_{2}(x)}(x)-\widetilde{u}(x)\right| \leq \frac{c}{2}$. Therefore, by (1.3) and because $\theta_{2}=0$ on a neighborhood of $\bar{D}_{1}$, the function $\widetilde{\psi}:=\widetilde{u}_{\delta \theta_{2}}$ has the required properties if $\delta>0$ is sufficiently small.

Now put $\psi:=\widetilde{\psi}-\frac{c}{2} \theta_{1}$ on $W$. We claim that

$$
\begin{gathered}
\psi \in \mathcal{S} \cap \mathrm{C}^{\infty}(W), \\
\psi \leq \widetilde{u} .
\end{gathered}
$$

Indeed, by (1.6), on $W \backslash \bar{D}_{1}$ we have $\psi=\widetilde{\psi}-\frac{c}{2} \leq \widetilde{u}$, and by $(1.5) \psi=\widetilde{u}-\frac{c}{2} \theta_{1}$ on a neighborhood of $W \cap \bar{D}_{1}$. Thus (1.8) follows, and from (1.4) we also get (1.7).

Define

$$
v:= \begin{cases}\max \{u, \psi\} & \text { on } W, \\ u & \text { on } \Omega \backslash W .\end{cases}
$$

From (1.8) we get that $v=u$ if $\eta=0$; thus from (1.2) and (1.7) it follows that $v \in \mathcal{S}(\Omega)$. Obviously i) is fulfilled, and (1.8) implies that $u \leq v \leq u+c$; hence ii) is also satisfied.

It remains to show iii). If $\eta=1$ then from (1.6) we obtain $\psi \geq \widetilde{u}-\frac{c}{2}-\frac{c}{2} \theta_{1} \geq u$; hence by (1.7) $v$ is smooth on a neighbborhood of $\bar{V}$. We claim that $v=u+c \eta$ on $G_{1}$; this will complete the proof. It is obvious outside $W$ (since $\eta=0$ there) and on $W \cap G_{1}$, by (1.5), we have $\psi=\widetilde{\psi}=u+c \eta$. The proof of the lemma is complete.

\section{Proof of Theorem B}

We shall say that $u$ is strictly convex in $\Omega$ if for every test function $\varphi \in \mathrm{C}_{0}^{\infty}(\Omega)$ the function $u+\varepsilon \varphi$ is convex in $\Omega$ for $\varepsilon>0$ small enough. This is equivalent to saying that locally one can find $\varepsilon>0$ such that $u(x)-\varepsilon|x|^{2}$ is convex. If $u$ is smooth then it is strictly convex if and only if the matrix $\left(\partial^{2} u / \partial x_{j} \partial x_{k}\right)$ is positive definite.

Results of the previous section give good approximation in the class of strictly convex functions:

Theorem 2.1. Let $\Omega$ and $\varepsilon$ be as in Theorem 1.1. Then for every strictly convex $u$ in $\Omega$ one can find a smooth, strictly convex $v$ in $\Omega$ such that $u \leq v \leq u+\varepsilon$. In particular, one can choose $v$ so that $\lim _{x \rightarrow \partial \Omega}(u(x)-v(x))=0$.

Proof. In view of Theorem 1.1 it is enough to prove the following

Proposition 2.2. The sheaf of strictly convex functions is a Richberg sheaf.

Proof. It is enough to show (1.3). Let $\Omega^{\prime}, \Omega, \theta$ and $u$ be as in (1.3). We can find an open $D \Subset \Omega$, a neighborhood of $\overline{\{\theta<1\}} \cap \overline{\Omega^{\prime}}$, such that for $\delta>0$ small enough $u_{\delta \theta}=u_{\delta}$ on a neighborhood of $\Omega^{\prime} \backslash D$ and $u_{\delta \theta}$ is smooth on $D$. We have

$$
\frac{\partial^{2} u(x-\delta \theta(x) y)}{\partial x_{j} \partial x_{k}}\left(x_{0}\right)=\frac{\partial^{2} u}{\partial x_{j} \partial x_{k}}\left(x_{0}-\delta \theta\left(x_{0}\right) y\right)+\delta \gamma_{j k}\left(x_{0}, y, \delta\right),
$$

where $\gamma_{j k}$ is uniformly bounded for $x_{0} \in D, y \in \bar{B}$ and $\delta \leq \delta_{0}$. This gives uniform convergence in $D$ of partial derivatives $\partial^{2} u_{\delta \theta} / \partial x_{j} \partial x_{k} \longrightarrow \partial^{2} u / \partial x_{j} \partial x_{k}$ as $\delta \downarrow 0$. Thus $u_{\delta \theta}$ is strictly convex on $\Omega^{\prime}$ for sufficiently small $\delta$, and the proposition follows. 
We are now in position to prove Theorem B.

Proof of Theorem B. Assume first that there is a smooth exhaustion $\psi$ such that every eigenvalue of the matrix $\left(\partial^{2} \psi / \partial x_{j} \partial x_{k}\right)$ is $\geq 1$. This means that every directional second derivative of $\psi$ is $\geq 1$. Suppose that the boundary of $D$ contains a line segment. Then approaching it by line segments lying in the interior we can easily get a contradiction.

Now let $D$ be strictly convex. Let $f$ be the restriction to $\partial D$ of the function $-2|x|^{2}$. We want to use Perron's method to extend $f$ to a function convex on $D$ and continuous on $\bar{D}$. By $u$ denote the supremum of all $v$ convex on $D$ and continuous on $\bar{D}$ with $v \leq f$ on $\partial D$. $u$ is of course convex on $D$ and continuous on $\bar{D}, u \leq f$ on $\partial D$, and we claim that $u=f$ on $\partial D$. Take $x_{0} \in \partial D$ and $\varepsilon>0$. We may assume that $x_{0}=0$ and $D$ lies in the half-space $\left\{x_{n}<0\right\}$. Then for $K$ big enough we have $K x_{n} \leq f(x)+\varepsilon$ on $\partial D$, thus $K x_{n}-\varepsilon \leq u(x)$. In particular $u(0) \geq-\varepsilon$, which proves the claim. Note that here $x_{n}$ is just a typical barrier function.

The function $\widetilde{u}(x):=u(x)+|x|^{2}$ is strictly convex; thus by Theorem 2.1 we can find a smooth, strictly convex $v$ with $v(x)=u(x)=-|x|^{2}$ on $\partial D$. Now it is enough to set $\psi(x):=v(x)+|x|^{2}$.

We can also prove a weaker version of Theorem A.

Theorem 2.3. In a bounded, convex domain there exists a smooth, strictly convex exhaustion.

Proof. Let $D$ be convex and bounded and let $u$ be any convex exhaustion in $D$. Put $v(x):=|x|^{2}-K$, where $K$ is so big that $v \leq 0$ in $D$. We claim that $\widetilde{u}:=-2(u v)^{1 / 2}$ is a strictly convex exhaustion. Indeed, in the case of smooth functions of one variable we have

$$
\left(-2(u v)^{1 / 2}\right)_{x x}=(u v)^{-1 / 2}\left(-u v_{x x}-v u_{x x}\right)+\frac{1}{2}(u v)^{-3 / 2}\left(u v_{x}-v u_{x}\right)^{2} \geq(u / v)^{1 / 2} v_{x x} ;
$$

thus in the general case

$$
\sum_{j, k=1}^{n} \frac{\partial^{2}(\widetilde{u}-\varepsilon v)}{\partial x_{j} \partial x_{k}} y_{j} y_{k} \geq\left((u / v)^{1 / 2}-\varepsilon\right)|y|^{2}, \quad y \in \mathbb{R}^{n}, \varepsilon>0 .
$$

This implies that $\widetilde{u}$ is strictly convex. Now it is enough to apply Theorem 2.1 to complete the proof.

\section{Preliminaries from the theory of the Monge-Ampère operator}

In this section we list properties of the Monge-Ampère operator which we shall use in section 4. A good reference is [RT]; see also [GT].

When $u$ is smooth, the Monge-Ampère operator takes the form

$$
M u:=\operatorname{det}\left(\frac{\partial^{2} u}{\partial x_{j} \partial x_{k}}\right) .
$$

If $u$ is convex then $M u$ can be defined by means of a gradient image as a nonnegative Borel measure, so that the following holds.

Theorem 3.1. If $\left\{u_{j}\right\}$ is a sequence of convex functions converging uniformly to $u$, then $M u_{j}$ tends weakly to $M u$. 
(Note that Theorem 3.1 can be regarded as an alternative definition of $M u$.)

The next property is sometimes called the comparison principle.

Theorem 3.2. Let $D$ be a bounded domain in $\mathbb{R}^{n}$ and let $u$ and $v$ be convex on $D$ and continuous on $\bar{D}$. Suppose that $u \leq v$ on $\partial D$ and $M u \geq M v$ in $D$. Then $u \leq v$ in $D$.

The next result solves the Dirichlet problem for the Monge-Ampère operator.

Theorem 3.3. Let $D$ be a bounded, strictly convex domain in $\mathbb{R}^{n}$, and suppose that $\mu$ is a nonnegative Borel measure on $D$ with $\mu(D)<+\infty$. Then for every continuous function $g$ on $\partial D$ there is a unique convex $u$ on $D$, continuous on $\bar{D}$, such that $M u=\mu$ in $D$ and $u=g$ on $\partial D$.

We shall also need the following fact.

Lemma 3.4. If $D$ is a convex domain in $\mathbb{R}^{n}$, then for every convex, continuous $u$ on $\bar{D}$ with $u \geq 0$ on $\partial D$ the following estimate holds:

$$
(-u(x))^{n} \leq c_{n}(\operatorname{diam} D)^{n-1} \operatorname{dist}(x, \partial D) M u(D), \quad x \in D,
$$

where $c_{n}$ is a constant depending only on $n$.

The following simple lemma makes it possible to express the Monge-Ampère operator (which is nonlinear) in terms of linear partial differential operators.

Lemma 3.5 [Gav]. By $\mathcal{A}$ denote the set of all symmetric, positive definite matrices $a$ with $\operatorname{det} a=1$. Then for any symmetric, positive semi-definite matrix $b$ one has

$$
(\operatorname{det} b)^{1 / n}=\frac{1}{n} \inf _{a \in \mathcal{A}} \operatorname{trace}(a b) .
$$

We will also need some local properties of the Monge-Ampère operator.

Proposition 3.6. For convex functions $u$ and $v$ one has

i) $M(u+v) \geq M u+M v$,

ii) $M \max \{u, v\} \geq 1_{\{u>v\}} M u+1_{\{u \leq v\}} M v$,

where $1_{A}$ stands for the characteristic function of a set $A$.

Proof. i) From Lemma 3.5 it follows that if matrices $b_{1}, b_{2}$ are symmetric and positive semi-definite, then

$$
\left(\operatorname{det}\left(b_{1}+b_{2}\right)\right)^{1 / n} \geq\left(\operatorname{det} b_{1}\right)^{1 / n}+\left(\operatorname{det} b_{2}\right)^{1 / n}
$$

which implies that $\operatorname{det}\left(b_{1}+b_{2}\right) \geq \operatorname{det} b_{1}+\operatorname{det} b_{2}$. Therefore we obtain i) for smooth $u, v$, and it is enough to apply Theorem 3.1 to get it for arbitrary $u$ and $v$.

ii) It is enough to show that for compact subsets $K$ of the set $\{u=v\}$ one has $M \max \{u, v\}(K) \geq M v(K)$. For $\delta>0$ put $v_{\delta}:=\max \{u, v+\delta\}$; then $v_{\delta}=v+\delta$ on a neighborhood of $K$ and $u_{\delta}$ decreases to $\max \{u, v\}$. From Theorem 3.1 we get

$$
M v(K)=\limsup _{\delta \rightarrow 0} M v_{\delta}(K) \leq M \max \{u, v\}(K) .
$$

Finally, the following result gives good regularity of solutions of the Dirichlet problem.

Theorem 3.7 [CNS]. Assume that $D$ is a smooth, bounded, strictly convex domain in $\mathbb{R}^{n}$. Take $\mu \in C^{\infty}(\bar{D})$ with $\mu>0$, and $g \in C^{\infty}(\partial D)$. Then the solution u given by Theorem 3.3 is smooth on $\bar{D}$. 


\section{Proof of Theorem A}

First we shall prove the following:

Theorem 4.1. Let $D$ be bounded, open and convex in $\mathbb{R}^{n}$, and let $\mu$ be a nonnegative Borel measure on $D$ with $\mu(D)<+\infty$. Then there exists a unique convex exhaustion $u$ in $D$ with $M u=\mu$.

Proof. The uniqueness follows immidiately from the comparison principle (Theorem 3.2). Assume first that $\mu$ is a continuous, compactly supported function in $D$. Let $\psi$ be a smooth, strictly convex exhaustion given by Theorem 2.3. Since $\mu$ has compact support and $\psi$ is strictly convex, we may assume that $M \psi \geq \mu$. Take strictly convex domains $D_{j} \uparrow D$. By Theorem 3.3 there exist convex exhaustions $u_{j}$ in $D_{j}$ such that $M u_{j}=\mu$ in $D_{j}$. From the comparison principle it follows that $\psi \leq u_{j+1} \leq u_{j}$ in $D_{j}$. Thus the sequence $\left\{u_{j}\right\}$ decreases to a convex exhaustion $u$ in $D$, and from Theorem 3.1 we obtain $M u=\mu$.

Now let $\mu$ be arbitrary. We can find a sequence $\left\{\mu_{j}\right\}$ of nonnegative continuous functions with compact supports in $D$, converging weakly to $\mu$ and such that $\mu_{j}(D) \leq A<+\infty$. From the previous part we can get convex exhaustions $u_{j}$ in $D$ with $M u_{j}=\mu_{j}$. By Lemma 3.4 the sequence $\left\{u_{j}\right\}$ is uniformly bounded on $D$. We can thus find a subsequence $\left\{u_{j_{k}}\right\}$ converging locally uniformly to some convex $u$ in $D$, and, again by Lemma $3.4, u$ is an exhaustion. Now it is enough to apply Theorem 3.1 to complete the proof.

We will use the above theorem with $\mu \equiv 1$. Note that if $D$ contains the origin and $\mu$ is the Dirac measure, then the exhaustion given by Theorem 4.1 is precisely $p_{D}-1$, where $p_{D}$ is the Minkowski functional of $D$.

Now we want to define a Richberg sheaf appropriate for Theorem A. First we prove one more local property of the Monge-Ampère operator.

Theorem 4.2. Let $u$ be a convex function with $M u \geq 1$. Then for every $\delta>0$ $M u_{\delta} \geq 1$, where $u_{\delta}:=u * \rho_{\delta}$ is the regularization of $u$.

Proof. From Lemma 3.5 it follows that for smooth, convex $v$ we have

$$
(M v)^{1 / n}=\inf _{a \in \mathcal{A}} \Delta_{a} v,
$$

where

$$
\Delta_{a}:=\frac{1}{n} \sum_{j, k=1}^{n} a_{j k} \frac{\partial^{2}}{\partial x_{j} \partial x_{k}} .
$$

We claim that to prove the theorem it is enough to show that $\Delta_{a} u \geq 1$ for $a \in \mathcal{A}$. Indeed, we would then have $\Delta_{a} u_{\delta}=\left(\Delta_{a} u\right) * \rho_{\delta} \geq 1$ and, by (4.1), $M u_{\delta} \geq 1$, which proves the claim.

For $\delta>0$ put $\mu_{\delta}:=M u * \rho_{\delta}$. Then $\mu_{\delta} \geq 1$, and we may assume that $u$ and $\mu_{\delta}$ are defined in a neighborhood of $\bar{B}$, where $B$ is a euclidean ball. Let $f^{\delta}$ be a sequence of smooth functions on $\partial B$, converging uniformly to $u$ there. From Theorem 3.7 we obtain a smooth, convex $u^{\delta}$ on $\bar{B}$ such that $M u^{\delta}=\mu_{\delta}$ in $B$ and $u^{\delta}=f^{\delta}$ on $\partial B$. From Proposition 3.6, part i), and Theorem 3.2 it follows that

$$
\tilde{u}+v^{\delta} \leq u^{\delta} \leq \tilde{u}-v^{\delta}
$$

where $\tilde{u}$ and $v^{\delta}$ are convex and continuous on $\bar{B}$ with $M \tilde{u}=0,\left.\tilde{u}\right|_{\partial B}=f$ and $M v^{\delta}=\mu_{\delta},\left.v^{\delta}\right|_{\partial B}=0$. By Lemma 3.4 the sequence $\left\{u^{\delta}\right\}$ is uniformly bounded 
on $B$; thus there exists a subsequence $\left\{u^{\delta_{j}}\right\}$ converging locally uniformly there. Moreover, (4.2) and Lemma 3.4 imply that $\left\{u^{\delta_{j}}\right\}$ is uniformly convergent on $\bar{B}$. From Theorem 3.2 it follows that $u^{\delta_{j}}$ converges to $u$. Since $u^{\delta_{j}} \in \mathrm{C}^{\infty}(\bar{B})$, from (4.1) we get $\Delta_{a} u^{\delta_{j}} \geq 1$. Now it is enough to observe that $\Delta_{a} u^{\delta_{j}}$ tends weakly to $\Delta_{a} u$; thus $\Delta_{a} u \geq 1$ and the theorem follows.

If $\Omega$ is open in $\mathbb{R}^{n}$, by $\mathcal{F}(\Omega)$ denote the set of all strictly convex $u$ in $\Omega$ with $M u>1$ (that is, for every $\Omega^{\prime} \Subset \Omega$ there exists $c>1$ such that $M u \geq c$ in $\Omega^{\prime}$ ).

Proposition 4.3. $\mathcal{F}$ defined above is a Richberg sheaf.

Proof. The definition of $\mathcal{F}$ is local, thus $\mathcal{F}$ is a sheaf. Take $u \in \mathcal{F}(\Omega), \varphi \in \mathrm{C}_{0}^{\infty}(\Omega)$ and $\Omega^{\prime} \Subset \Omega$. We may assume that $\operatorname{supp} \varphi \subset \Omega^{\prime}$. If $M u \geq c>b>1$ in $\Omega^{\prime}$, then by Proposition 3.6, part i), for $\varepsilon>0$ small enough we have

$$
M(u+\varepsilon \varphi) \geq M\left(b^{-1 / n} u\right) \geq c / b>1,
$$

because $\left(1-b^{-1 / n}\right) u$ is strictly convex. This implies that $\mathcal{F}$ satisfies (1.1). Proposition 3.6, part ii), implies that $\mathcal{F}$ also satisfies (1.2).

From Theorem 4.2 it follows that $\mathcal{F}$ satisfies (1.3)'. Let $\Omega^{\prime}, \Omega, \theta$ and $u$ be as in (1.3). If $D$ is as in the proof of Proposition 2.2, in the same way as there we can get uniform convergence of the partial derivatives $\partial^{2} u_{\delta \theta} / \partial x_{j} \partial x_{k} \longrightarrow \partial^{2} u / \partial x_{j} \partial x_{k}$ as $\delta \downarrow 0$ on $D$, whereas on a neighborhood of $\Omega^{\prime} \backslash D$ we have $u_{\delta \theta}=u_{\delta}$ for $\delta>0$ small enough. The proof of the proposition is complete.

Proof of Theorem A. In view of Proposition 4.3 and Theorem 1.1 it is enough to show the existence of a strictly convex exhaustion $u$ with $M u>1$. But this is obvious, thanks to Theorems 2.3, 4.2 and Proposition 3.6, part i).

\section{REFERENCES}

[Bło] Z.Błocki, The complex Monge-Ampère operator in hyperconvex domains, Ann. Scuola Norm. Sup. Pisa (to appear).

[CNS] L. Caffarelli, L. Nirenberg, J.Spruck, The Dirichlet problem for nonlinear second order elliptic equations. I. Monge-Ampère equations, Comm. Pure Appl. Math. 37 (1984), 369402. MR 87f:35096

[Gav] B. Gaveau, Méthodes de contrôle optimal en analyse complexe. I. Résolution d'équations de Monge-Ampère, J. Funct. Anal. 25 (1977), 391-411. MR 56:15987

[GT] D. Gilbarg, N. S. Trudinger, Elliptic partial differential equations of second order, Grundl. d. math. Wiss. 244, Springer-Verlag, 1983. MR 86c:35035

[Hör] L. Hörmander, Notions of convexity, Birkhäuser, 1994. MR 95k:00002

[KR] N. Kerzman, J.-P. Rosay, Fonctions plurisousharmoniques d'exhaustion bornées et domaines taut, Math. Ann. 257 (1981), 171-184. MR 83g:32019

[RT] J.Rauch, B. A. Taylor, The Dirichlet problem for the multidimensional Monge-Ampère equation, Rocky Mountain Math. J. 7 (1977), 345-364. MR 56:12582

[Rich] R. Richberg, Stetige streng pseudokonvexe Funktionen, Math. Ann. 175 (1968), 257-286. MR 36:5386

[Sib] N. Sibony, Une classe de domaines pseudoconvexes, Duke Math. J. 55 (1987), 299-319. MR 88g:32036

Jagiellonian University, Institute of Mathematics, Reymonta 4, 30-059 Kraków, POLAND

E-mail address: blocki@im.uj.edu.pl 